\title{
Review of: "Simplifying the dental/periodontal management of patients with metabolic bone fragility receiving treatment with denosumab"
}

\author{
Francesco Maria Erovigni ${ }^{1}$
}

1 University of Turin

Potential competing interests: The author(s) declared that no potential competing interests exist.

I read this manuscript with great interest, the purpose of research is certainly interesting and topical. The article proposes a treatment protocol for elective dental surgery (secondary prevention) based on pharmacokinetic of denosumab (DNS) in patients affected from metabolic bone disorders. The article focuses on patients affected from metabolic bone disorders whose have started antiresorptive therapy with DNS first.

It would be interesting to know the rating of this subset among the entire population undergoing AR therapy, since for a great part of the osteoporotic population a BPs or N-BPs oral/intramuscular therapy is usually the first choice of treatment and DNS is suggested only after a worsening of osteoporosis. Besides, I would like to know how to consider patients at increased risk of fragility fractures who are shifted from oral/im NBP to DNB treatment with a time of cumulative dosage lower than 3 years for NBP.

The article is well written, presents good reflections and gives clear indications for the invasive oral treatment of patients with metabolic bone fragility receiving DNS. 